Mamoru K.

Nagoya Math. J.

Vol. 33 (1968) 21-52

\title{
ON THE SINGULARITY OF GREEN FUNCTIONS IN MARKOV PROCESSES
}

\author{
MAMORU KANDA
}

\section{§0. Introduction}

In the previous paper [6] we have discussed Markov processes in $R^{d}$ with the Green function $G(x, y)$ satisfying $C_{1} \frac{1}{|x-y|^{d-\alpha}} \leq G(x, y)$ $\leq C_{2} \frac{1}{|x-y|^{-\alpha}}\left(0<\alpha \leqq 2, C_{1}<C_{2}\right.$ are positive constants), and showed that the regular points of its process are the same as those of $\alpha$-stable process. The present article is closely related to the previous one. We shall discuss several properties of Markov process, including those of regular points, which are sharply influenced by the singularity of the Green function at diagonal set. The singularity we will be concerned with is more general than that of previous one, but it is closely related to that of Riesz kernel. Then, our results may be considered as a generalization of the facts which appear in the relation between the Riesz kernel and stable process. For this purpose potential representation of the hitting probability plays an important role, which we shall show under certain uniform condition about singularity of the Green function instead of duality condition.

The author wishes his thanks to Prof. H. Kunita for his valuable suggestions.

\section{§1. Notations and main results}

Let $R^{d}(d \geq 3)$ be the $d$-dimensional Euclidean space and $\Omega$ be a domain in it. Let $\infty$ be adjoined to $\Omega$ and $\Omega \cup\{\infty\}$ be its one-point compactification. We denote by $\mathscr{B}$ the topological Borel field on $\Omega \cup\{\infty\}$. We introduce several spaces of functions on $\Omega ; B_{K}=$ the space of bounded $\mathscr{B}$ measurable functions of compact support, $C_{K}=$ the space of continuous functions of compact support in $\Omega$ and $C_{0}=$ the space of continuous functions vanishing at infinity.

An extended real-valued function $G(x, y)$ on $\Omega \times \Omega$ is said to be a kernel on $\Omega$, if it is non-negative, continuous except at the diagonal set on $\Omega \times \Omega$.

Received Nov. 10, 1967. 
Further we assume that $G$ maps $B_{K}$ into $C_{0}$, where $G f(x)=\int_{\Omega} G(x, y) f(y) d y$. We say that a kernel $G(x, y)$ on $\Omega$ satisfies the complete maximum principle, if for any constant $a \geqq 0, f$ and $g \in C_{K}^{+}$, the inequality

$$
G f(x) \leqq G g(x)+a
$$

holds everywhere, if it holds on the support of $f$, where $C_{K}^{+}$denotes the set of non-negative functions of $C_{K}$.

In this article we consider a kernel $G(x, y)$ which has singularity $\varphi(|x-y|)$ at $x=y$, more precisely, for each $x_{0}$ there exists a ball $0_{x_{0}}$ centering at $x_{0}$ and

$$
G(x, y)=C(x, y) \varphi(|x-y|),
$$

where $C(x, y)$ is bounded on $\bar{O}_{x_{0}} \times \bar{O}_{x_{0}}$ and $\inf _{x, y \in \bar{O}_{x_{0}}} C(x, y)>0$, where $|x-y|$ denotes the distance between $x$ and $y$ and $\bar{O}_{x_{0}}$ denotes the closure of $O_{x_{0}}$. In this case we say that $G(x, y)$ is a kernel with singularity $\varphi$. We always choose a function $\varphi$ which shows the singularity of a kernel from one of the following classes;

$\Phi=$ the space of non-negative, continuous and monotone decreasing functions $\varphi(t)$ defined for sufficiently small $t>0$ such that $\lim _{t \rightarrow 0} \varphi(t)=+\infty, \int_{0} t^{d-1} \varphi(t) d t<+\infty$, $\Phi_{1}=$ the space of functions $\varphi(t) \in \Phi$ for which there exists an integer $p, 0<p<d$, such that $t^{p} \varphi(t)$ is monotone increasing for sufficiently small $t>0$ and $\lim _{t \rightarrow O} t^{p} \varphi(t)=0$,

$\Phi_{1}^{M}=$ the space of functions $\varphi(t)$ defined for all $t>0$ and of the class $\Phi_{1}$ such that, if we set $G(x, y)=\varphi(|x-y|), G$ is a kernel on $R^{d}$ satisfying the complete maximum principle.

Now we shall prepare the notations of Markov processes. Let $\omega$ be a function $[0,+\infty) \rightarrow \Omega \cup\{\infty\}$ such that it has right-continuity and left hand limits everywhere and if $\omega(t)=\infty$, then $\omega\left(t^{\prime}\right)=\infty$ for all $t^{\prime} \geqq t$. Let us set $\zeta(\omega)=\inf \{t \geqq 0, \omega(t)=\infty\},=+\infty$ if there are no such $t$. Let $W$ be a set of $\omega$ 's mentioned above. Then $W$ is closed under the operation of the shift; $\omega \rightarrow \theta_{t} \omega, t \geqq 0$, where $\left(\theta_{t} \omega\right)(s)=\omega(t+s) . \quad F$ is the $\sigma$-field in $W$ generated by sets $\{\omega(t) \in A\}, t \geqq 0, A \in \mathscr{B}$, and $F_{t}$ is the $\sigma$-field in $W_{t}=\{\zeta>t\}$ generated by the sets $\{\omega(s) \in A, \zeta>t\}, 0 \leqq s \leqq t, A \in \mathscr{B}$. Let $\left\{P_{x}, x \in \Omega\right\}$ be a family of probability measures on $(W, F)$ such that $P_{x}(B)$ is $\mathscr{B}$-measurable in $x$ for each fixed $B \in F$. We denote by $M$ the intersection of all 
$P_{\mu}$-completed $\sigma$-fields of $F$, where $P_{\mu}(\cdot)$ denotes the measure defined by $\int_{\Omega} P_{x}(\cdot) \mu(d x)$ for any measure $\mu$ and, by $M_{t}$ the $\sigma$-field formed from $F_{t}$ and all null sets of $M\left[W_{t}\right]$, where $M\left[W_{t}\right]$ is the restriction of $M$ to $W_{t}$. The system $X=\left(x_{t}, \zeta, M_{t}, P_{x}\right)$ is called a Hunt process if it has the strong Markov property and the quasi-left continuity of sample functions. A Hunt process $X=\left(x_{t}, \zeta, M_{t}, P_{x}\right)$ is called a Feller process if its semi-group $\left\{T_{t}\right\}$ is a strongly continuous operator on $C_{0}$. For a nearly analytic set $E$, we define the hitting time by $\sigma_{E}=\inf \left(t>0, x_{t} \in E\right),=+\infty$ if there are no such $t$. Then $\sigma_{E}$ is a Markov time and we call $P_{x}\left(\sigma_{E}<+\infty\right)$ the hitting probability of $E$.

A kernel $G(x, y)$ on $\Omega$ is called the Green function of a Feller process $X=\left(x_{t}, \zeta, M_{t}, P_{x}\right)$ on $\Omega$ if it satisfies

$$
E_{x}\left\{\int_{0}^{\zeta} f\left(x_{t}\right) d t\right\}=\int_{\Omega} G(x, y) f(y) d y
$$

for every function $f$ of $B_{K}$. The Green function $G(x, y)$ is called quasisymmetric, if $\hat{G}(x, y) \equiv G(y, x)$ is a kernel on $\Omega$ which satisfies the complete maximum principle.

In $\S 2$ we shall study the following potential representation theorem of the hitting probability, which has been studied in the works of Ito-Mckean [5], G.A. Hunt [4], M.G. Sur [14] and H. Kunita-T. Watanabe [10], etc. In their works it seems that some condition about duality is necessary intrinsically. Here we shall show that, if we require certain uniform condition about the singularity of the Green function instead of the duality condition, the method of representing the hitting probability as a potential discussed in H. Kunita and T. Watanabe [10], is applicable with some modifications. Then we have the following

Theorem 1. Let $X=\left(x_{t}, \zeta, M_{t}, P_{x}\right)$ be a Feller process on $\Omega$ which has the Green function $G(x, y)$ with singularity $\varphi \in \Phi$. Let $K$ be a nearly analytic set with compact closure in $\Omega$. If we assume the condition $B$, (see the supplement),

i) then there exists a measure $\mu_{K}$ whose support is included in $\bar{K}$ such that

$$
P_{x}\left(\sigma_{K}<+\infty\right)=\int_{\Omega} G(x, y) \mu_{K}(d y)
$$

for any $x \in \Omega$, where $\bar{K}$ denotes the closure of $K$.

ii) Furthermore, the measure $\mu_{K}$ is unique if one of the followings are satisfied:

a) $X$ is a continuous process, 
b) $G(x, y)$ is quasi-symmetric.

In $\S 3$ we construct a Feller process on $\Omega$ whose Green function is a given kernel satisfying the complete maximum principle with singularity $\varphi \in \Phi$ (Theorem 2).

$\S 4$ is devoted to studying some properties of capacitary measure $\mu_{K}(d y)$ in case the process $X$ has the quasi-symmetric Green function. Most of these are modifications of Hunt's results [4] to our setting and these are used in the later discussions.

In the remaining sections we show some applications of Theorem 1 and 2. O. Frostman [3] has shown that the Riesz kernel with exponent $\alpha>2$ does not satisfy the maximum principle. This corresponds to the fact that $e^{\text {-const. }|z|^{\alpha}}, \alpha>2$, cannot be a characteristic function of a distribution function. In $\S 5$ we extend this fact to a kernel with singularity $\varphi \in \Phi$ in the following form by using Theorem 1 and 2 .

Theorem 3. Let $G(x, y)$ be a kernel with singularity $\varphi \in \Phi$ on $\Omega$ and let $C(x, y)\left(=\frac{G(x, y)}{\varphi(|x-y|)}\right)$ can be considered as a continuous function on a suitable neighborhood of each diagonal point. Then, if $G(x, y)$ is a quasi-symmetric Green function it is necessary for $\varphi(t)$ to satisfy the following inequality

$$
\varphi(r)<\frac{M_{1}}{\sqrt{\pi}} \int_{0}^{\pi} \varphi\left(2 r \sin \frac{\theta}{2}\right) \sin ^{d-2} \theta d \theta
$$

for each sufficiently small $r>0$ and each constant $M_{1}>\Gamma\left(\frac{d}{2}\right) \Gamma\left(\frac{d-1}{2}\right)^{-1}$.

In the case when the singularity function $\varphi$ satisfies the above inequality for $M_{1}=\Gamma\left(\frac{d}{2}\right) \Gamma\left(\frac{d-1}{2}\right)^{-1}$, we cannot say, in general, whether the theorem is valid or not. But if we assume that the Green function $G(x, y)$ with singularity $\varphi$ is written as the difference of $x$ and $y$, the above theorem is still valid even for $M_{1}=\Gamma\left(\frac{d}{2}\right) \Gamma\left(\frac{d-1}{2}\right)^{-1}$.

It is well known that a Feller process corresponding to the Riesz kernel with exponent $\alpha$ (stable process) is not a continuous process in case $0<\alpha<2$. In $\S 6$, we shall extend this fact to the following form.

Theorem 4. Let $X$ be a Feller process having the Green function which satisfies the same assumptions as those of Theorem 3. If it holds the following inequality 


$$
\varphi(r)<\frac{M_{2}}{\sqrt{\pi}} \int_{0}^{\pi} \varphi\left(2 r \sin \frac{\theta}{2}\right) \sin ^{d-2} \theta d \theta<+\infty
$$

for sufficiently small $r$, where $M_{2}$ is a positive constant strictly smaller than $\Gamma\left(\frac{d}{2}\right) \Gamma\left(\frac{d-1}{2}\right)^{-1}, X$ is not a continuous process.

The above Theorem will conclude naturally that any Feller process with singularity $t^{\alpha-d}, 0<\alpha<2$, or, more generally, $t^{\alpha-d}\left(\log \frac{1}{t}\right)^{k}, 0<\alpha<2$, is not a continuous process under the continuity assumption on $C(x, y)$.

We shall give a theorem about regular points which is an improvement of our previous result(*) [6] and will be proved in $\$ 7$.

Theorem 5. Let $X^{1}=\left(x_{t}^{1}, \zeta^{1}, M_{t}^{1}, P_{x}^{2}\right)$ and $X^{2}=\left(x_{t}^{2}, \zeta^{2}, M_{t}^{2}, P_{x}^{2}\right)$ be Feller processes on $\Omega$ having the Green function $G^{1}(x, y)$ with singularity $\varphi_{1}$ of $\Phi_{1}$ and, $G^{2}(x, y)$ with singularity $\varphi_{2}$ of $\Phi_{1}$, respectively. Suppose that $\frac{\varphi_{1}(t)}{\varphi_{2}(t)}$ is monotone non-decreasing for sufficiently small $t$. If we assume the condition $B$ for $X^{1}$ and $X^{2}$,

i) then, a point $x_{0}$ is a regular point of $B$ for $X^{1}$, if it is a regular point of $B$ for $X^{2}$, where $B$ is a compact set.

ii) Further, suppose that $\varphi_{1}$ and $\varphi_{2}$ belong to the class $\Phi_{1}^{M}$ and $\frac{\varphi_{1}(t)}{\varphi_{2}(t)}$ decreases to zero as $t$ tends to zero. Then the regular points of $B$ for $X^{2}$ are included in the regular points of $B$ for $X^{1}$ in the strict sense, more precisely, there exists a compact set $B$ such that some points of $B$ are regular points of $B$ for $X^{1}$ but no point of $B$ is a regular point of $B$ for $X^{2}$.

The above theorem is applied to the (quasi-) diffusion corresponding to the strictly elliptic differential operator of the second order with Dini continuous coefficients in the strict sense.

We shall finally give an example of Feller process in $R^{3}$ whose Green function is a kernel with singularity $t^{\alpha-3} \log \frac{1}{t}, 1<\alpha \leqq 2$ in $\S 8$.

\section{\$2. Potential representation of the hitting probability}

Throughout this section we shall consider a Feller process $X=\left(x_{t}, \zeta, M_{t}, P_{x}\right)$ which has the Green function $G(x, y)$ with singularity $\varphi \in \Phi$ without special mentioning. Let us set $G_{\alpha} f(x)=\int_{0}^{+\infty} e^{-\alpha t} T_{t} f(x) d t$ for each $\alpha \geqq 0$. Then

*) We have to point out the incorrectness in our previous paper [6]. In the formula (4.19) of [6], "analytic set $E$ " should be replaced by "compact set $E$ ". Hence Theorem 4.2 of [6] is true for a compact set. 
$\left\{G_{\alpha}\right\}$ is a resolvent on $C_{0}$ such that $\lim _{\alpha \rightarrow+\infty} \alpha G_{\alpha} f=f$ for each bounded continuous function $f$. A positive universally measurable function $u$ is said to be $(G, \alpha)$-excessive if $\beta G_{\alpha+\beta} u \leqq u$ for all $\beta>0$ and if $\lim _{\beta \rightarrow+\infty} \beta G_{\alpha+\beta} u=u$.

We shall first prepare some lemmas for the proof of Theorem 1 .

Lemma 1. $G(x, y)$ is a $(G, 0)$-excessive function of $x$ for each fixed $y$.

This is shown in [7].

Remark. Let $\mu$ be a measure such that $u(x)=\int_{\Omega} G(x, y) \mu(d y)$ is bounded. Then $u(x)$ is superharmonic in $\Omega$, that is, for each domain $Q \subset \Omega, E_{x} u\left(x_{\tau_{Q}}\right)$ $\leqq u(x)$, where $\tau_{Q}=\inf \left(t \geqq 0 ; x_{t} \notin Q\right)$.

Lemma 2. Let $K$ be a nearly analytic set with compact closure. Then $P_{x}\left(\sigma_{K}<+\infty\right)$ is $(G, 0)$-excessive and further it is harmonic in $\Omega-\bar{K}$, that is, for each domain $Q \subset \Omega-\bar{K}, E_{x}\left(P_{x_{\tau_{Q}}}\left(\sigma_{K}<+\infty\right)\right)=P_{x}\left(\sigma_{K}<+\infty\right)$, under the condition $B$.

Proof. This is a direct consequence of the strong Markov property.

Lemma 3. Let $\left\{G_{n}\right\}$ be a sequence of compact sets such that $G_{n} \uparrow \Omega, G_{n} \supset K$ for each $n$. Then we have, under the condition $B$,

$$
\lim _{n \rightarrow+\infty} E_{x}\left(P_{x_{\sigma_{\sigma_{n}^{c}}}}\left(\sigma_{K}<+\infty\right)\right)=0 .
$$

Proof. We shall prove this in the same way as Lemma 3 in M.G. Sur [14]. Noting that $E_{x}\left(P_{x_{\sigma_{\sigma_{l}}}}\left(\sigma_{K}<+\infty\right)\right)=E_{x}\left(P_{x}\left(\sigma_{K}\left(\omega_{\sigma_{\sigma_{l}}}^{+}\right)<+\infty\right)\right)=P_{x}\left(\sigma_{K}<+\infty\right)$, we have for $n>l$

$$
\begin{aligned}
& E_{x}\left(P_{x_{\sigma_{G}^{c}}^{c}}\left(\sigma_{K}<+\infty\right)\right)=E_{x}\left\{E_{x_{\sigma_{\sigma_{n}^{c}}}}\left(P_{x_{\sigma_{G_{l}}}}\left(\sigma_{K}<+\infty\right)\right)\right\} \\
& =E_{x}\left\{E_{x}\left(P_{x_{\sigma_{G_{l}}}}\left(\omega_{\sigma_{G_{n}^{c}}^{+}}\right)\left(\sigma_{K}<+\infty\right)\right)\right\} \leqq P_{x}\left(\sigma_{G_{n}^{c}}+\sigma_{G_{l}}\left(\omega_{\sigma_{G_{n}^{c}}}^{+}\right)<+\infty\right) \\
& =P_{x}\left(\sigma_{G_{n}^{c}}+\sigma_{G_{l}}\left(\omega_{\sigma_{\sigma_{n}^{c}}}^{+}\right)<\zeta\right), \quad \theta_{t} \omega \equiv \omega_{t}^{+} .
\end{aligned}
$$

On the other hand, let $\xi_{n}(w) \equiv \sigma_{G_{n}^{c}}+\sigma_{G_{l}}\left(\omega_{\sigma_{n}^{c}}^{+}\right)$, we have

$$
E_{x}\left\{\int_{G_{l+1}} G\left(x_{\xi_{n}}, y\right) d y\right\} \geqq \delta \circ P_{x}\left(\xi_{n}<\zeta\right)
$$

where $\delta=\min _{x \in \bar{G}_{l}} \int_{G_{l+1}} G(x, y) d y>0 . \quad\left(\right.$ Since $\int_{G_{l+1}} G(x, y) d y$ is continuous in $\bar{G}_{l} \subset G_{l+1}$, $\min _{x \in \bar{G}_{l}} \int_{G_{l+1}} G(x, y) d y$ is attained at some point $x_{0}$ of $\bar{G}_{l}$. It is clear that 
$\int_{G_{l+1}} G\left(x_{0}, y\right) d y>0$.) We have also

$$
\begin{aligned}
& E_{x}\left\{\int_{G_{l+1}} G\left(x_{\xi_{n}}, y\right) d y\right\}=E_{x}\left\{\int_{\xi_{n}}^{\zeta} \chi_{G_{l+1}}\left(x_{t}\right) d t\right\} \leqq E_{x}\left\{\int_{\sigma_{G_{n}^{c}}^{c}}^{\zeta} \chi_{G_{l+1}}\left(x_{t}\right) d t\right\} \\
& \quad=E_{x}\left\{\int_{\Omega} G\left(x_{\sigma_{G_{n}^{c}}^{c}}, y\right) \chi_{G_{l+1}}(y) d y\right\},
\end{aligned}
$$

where $\chi_{G_{l+1}}$ is a characteristic function of $G_{l+1}$. Noting that $G$ maps $B_{K}$ into $C_{0}$, the last expression tends to zero as $n \rightarrow+\infty$, which implies $P_{x}\left(\xi_{n}<\zeta\right) \rightarrow 0$. Hence $\lim _{n \rightarrow \infty} E_{x}\left(P_{x_{\sigma_{G_{n}^{c}}}}\left(\sigma_{K}<+\infty\right)\right)=0$.

Lemma 4. Under the same assumption in Lemma 3, we have

$$
E_{x} G\left(x_{\sigma_{G_{n}^{e}}}, y\right)=G(x, y)
$$

for almost all $y$ in $G_{n}^{c}$.

Proof. Let $f$ be any function of $B_{K}$ vanishing in $G_{n}$. Then we have

$$
\begin{aligned}
\int_{\Omega} E_{x} G\left(x_{\sigma_{G_{n}^{e}}}, y\right) f(y) d y & =E_{x}\left\{E_{x_{\sigma_{\sigma_{n}^{e}}}}\left(\int_{0}^{+\infty} f\left(x_{t}\right) d t\right)=E_{x}\left\{\int_{\sigma_{\sigma_{n}^{e}}^{e}}^{+\infty} f\left(x_{t}\right) d t\right\}\right. \\
=E_{x}\left\{\int_{0}^{+\infty} f\left(x_{t}\right) d t\right\} & =\int_{\Omega} G(x, y) f(y) d y
\end{aligned}
$$

for each $x$ in $\Omega$. Hence we complete the proof.

Lemma 5. Let us fix a point $x_{0}$ and a ball $V$ containing $x_{0}$. Then there exists a ball $U\left(x_{0}\right)$ which centers at $x_{0}$ with $U\left(x_{0}\right) \subset V$ such that it holds

$$
G(x, y)>E_{x} G\left(x_{\tau_{V}}, y\right)
$$

for each $(x, y) \in U\left(x_{0}\right) \times U\left(x_{0}\right)$, where $\tau_{V}=\inf \left(t \geqq 0 ; x_{t} \notin V\right)$.

Proof. If we choose a ball $U\left(x_{0}\right)$ such that $U\left(x_{0}\right) \subset O_{x_{0}} \cap V$ we have $G(x, y) \geqq \inf _{\left(x^{\prime}, y^{\prime}\right) \in O_{x_{0}} \times O_{x_{0}}} C\left(x^{\prime}, y^{\prime}\right) \cdot \varphi\left(\right.$ diameter of $\left.U\left(x_{0}\right)\right)$ for each $(x, y) \in U\left(x_{0}\right)$ $\times U\left(x_{0}\right)$, and $E_{x} G\left(x_{\tau_{v}}, y\right) \leqq \sup _{y^{\prime} \in U\left(x_{0}\right), x^{\prime} \in V^{c}} G\left(x^{\prime}, y^{\prime}\right)$. Hence the lemma holds if the diameter of $U\left(x_{0}\right)$ is sufficiently small.

Proof of (i) of theorem 1. We shall first show that there exists a positive measure $\mu_{K}(d y)$ such that

$$
P_{x}\left(\sigma_{K}<+\infty\right)=\int_{\Omega} G(x, y) \mu_{K}(d y)
$$

holds everywhere on the similar way as in the proof of Prop. 7. 6. in H. 
Kunita and T. Watanabe [10]. Let us set $u(x)=P_{x}\left(\sigma_{K}<+\infty\right)$ and $u_{n}=n G_{n} u$, where $G_{n} u(x)=E_{x}\left(\int_{0}^{+\infty} e^{-n t} u\left(x_{t}\right) d t\right)$. Then $u_{n}$ increases to $u$ with $n$, because $u$ is a $(G, O)$-excessive functions, and by using Dynkin's formula and noting Lemma 3 , we have $u_{n}(x)=G f_{n}(x)$, where $f_{n}(x)=n\left(u(x)-u_{n}(x)\right)$. As $G f_{n}(x) \leqq 1$ and $\inf _{y \in A} \int_{B} G(x, y) d x>\delta_{B}>0$ for each compact set $A, B$ such that $A \subset A^{\prime} \subset \stackrel{\circ}{B}, \stackrel{\circ}{B} \neq \phi$, where $\stackrel{\circ}{B}$ denotes the set of interior point of $B$ and $A^{\prime}$ is a fixed compact set in $\stackrel{\circ}{B}$, we have

$$
V(B) \geqq \int_{B} d x \int_{A} G(x, y) f_{n}(y) d y \geqq \delta_{B} \int_{A} f_{n}(y) d y,
$$

where $V(B)$ is the volume of $B$. Therefore there exists a subsequence of measures $\left\{f_{n_{k}}(x) d x\right\}$ and a measure $\mu_{K}(d x)$ such that $\lim _{k \rightarrow+\infty} \int_{\Omega} g(x) f_{n_{k}}(x) d x$ $=\int_{\Omega} g(x) \mu_{K}(d x)$ for each $g \in C_{K}$. Now, let $h$ be a positive function of $B_{K}$, then, noting that $\hat{G} h(x)\left(=\int_{\Omega} \hat{G}(x, y) h(y) d y, \hat{G}(x, y)=G(y, x)\right)$ is continuous, we have

$$
\begin{aligned}
& \int_{\Omega} h(x) u(x) d x=\lim _{k \rightarrow+\infty} \int_{\Omega} h(x) G f_{n_{k}}(x) d x \\
& \geqq \int_{\Omega} \hat{G} h(x) \mu_{K}(d x)=\int_{\Omega} G \mu_{K}(x) h(x) d x, G \mu_{K}(x)=\int_{\Omega} G(x, y) \mu_{K}(d y) .
\end{aligned}
$$

Let $G_{n}$ be a sequence of compact sets mentioned in Lemma 3, then we have

$$
\begin{aligned}
\int_{\Omega} h(x) u(x) d x & =\lim _{k \rightarrow+\infty} \int_{\Omega} h(x) G f_{n_{k}}(x) d x \\
& \leqq \lim _{k \rightarrow+\infty} \int_{\Omega} h(x) \int_{G_{n}} G(x, y) f_{n_{k}}(y) d y d x \\
& +\lim _{k \rightarrow+\infty} \int_{\Omega} h(x) \int_{G_{n}^{c}} G(x, y) f_{n_{k}}(y) d y d x
\end{aligned}
$$

Noting Lemma 4, we have

$$
\begin{aligned}
& \text { the right-hand side of the above equality } \leqq \int_{\Omega} G \mu_{K}(y) h(y) d y \\
& +\lim _{k \rightarrow+\infty} \int_{\Omega} h(x) d x \int_{G_{n}^{c}} E_{x} G\left(x_{\sigma_{G_{n}^{c}}}, y\right) f_{n_{k}}(y) d y \\
& \leqq \int_{\Omega} G \mu_{K}(y) h(y) d y+\int_{\Omega} E_{x} u\left(x_{\sigma_{n}^{c}}\right) h(x) d x .
\end{aligned}
$$


Therefore by Lemma 3, we have

$$
\int_{\Omega} h(x) u(x) d x \leqq \int_{\Omega} G \mu_{K}(y) h(y) d y .
$$

Hence we have by (2.1) and (2. 2)

$$
G \mu_{K}(x)=u(x) \text {, almost everywhere. }
$$

Since $G \mu_{K}(x)$ and $u(x)$ are $(G, O)$-excessive, the equality holds everywhere.

We shall next show that $\mu_{K}$ concentrates on $\bar{K}$. Let $A$ be a compact set in $K^{c}$, it is sufficient to show that $\mu_{K}(A)=0$. Now we shall assume that $\mu_{K}(A)>0$. Let $x_{0}$ be a point of $A$ such that for every neighborhood $Q$ of $x_{0} \mu_{K}(Q)>0$ and let us choose a ball $V \subset \bar{K}^{c}$ whose center is $x_{0}$ and fix them, then we can choose a ball $U\left(x_{0}\right)$ which centers at $x_{0}$ such that the inequality of Lemma 5 holds for each $(x, y) \in U\left(x_{0}\right) \times U\left(x_{0}\right)$. Therefore we have

$$
u_{1}\left(x_{0}\right) \equiv \int_{U\left(x_{0}\right) \cap A} G\left(x_{0}, y\right) \mu_{K}(d y)>E_{x_{0}}\left\{\int_{U\left(x_{0}\right) \cap A} G\left(x_{\tau_{V}}, y\right) \mu_{K}(d y)\right\}
$$

and noting that $u_{2}\left(x_{0}\right) \equiv \int_{\Omega-U\left(x_{0}\right) \cap A} G\left(x_{0}, y\right) \mu_{K}(d y) \geqq E_{x_{0}} u_{2}\left(x \tau_{V}\right)$,

$$
u\left(x_{0}\right)=u_{1}\left(x_{0}\right)+u_{2}\left(x_{0}\right)>E_{x_{0}}\left\{u_{1}\left(x_{\tau_{V}}\right)+u_{2}\left(x_{\tau_{v}}\right)\right\}=E_{x_{0}} u\left(x_{\tau_{v}}\right),
$$

which contradicts the fact that $u(x)$ is harmonic in $\Omega-\bar{K}$. Hence $\mu_{K}(A)=0$.

Generally it is open whether the measure $\mu_{K}(d y)$ is uniquely determined or not. But in case the process is continuous we can show the uniqueness of $\mu_{K}(d y)$. Before the proof we prepare a lemma.

Lemma 6. Let $\mu$ be a positive measure such that $G \mu$ is bounded and $E$ be a nearly analytic set in $\Omega$. Then $\int_{\bar{E}} G(x, y) \mu(d y)$ is harmonic in $\Omega-\bar{E}$, if the process $X$ is continuous.

Proof. Let $Q$ be an open set in $\Omega-\bar{E}$. By the continuity of the path we have $P_{x}\left(x \tau_{Q} \in \partial Q \cup\{\infty\}\right)=1$, so $E_{x} G\left(x_{\tau_{Q}}, y\right)$ is continuous in $\Omega-\bar{Q}$ with respect to $y$, where $x$ is in the interior of $Q$. Hence $E_{x} G\left(x_{\tau_{Q}}, y\right)=G(x, y)$ holds for every points $y$ of $E$, because it holds for almost all $y$ in $\bar{Q}^{c}$. (See Lemma 4).

The first half of proof of theorem 1, (ii). We assume the condition (a). Let $\mu_{K}^{1}(d y)$ and $\mu_{K}^{2}(d y)$ be measures concentrating on $\bar{K}$ such that 
$u(x)=\int_{\Omega} G(x, y) \mu_{K}^{1}(d y)=\int_{\Omega} G(x, y) \mu_{K}^{2}(d y)$ for all $x$. It is sufficient to show that for each open set $\Omega \supset Q$ we have

$$
\int_{Q} G(x, y) \mu_{K}^{1}(d y)=\int_{Q} G(x, y) \mu_{K}^{2}(d y)
$$

Let $C$ be a compact set in $Q$ and let us set $v(x)=\int_{Q} G(x, y) \mu_{K}^{1}(d y)$ $-\int_{C} G(x, y) \mu_{K}^{2}(d y)$, then $v(x)$ is superharmonic in $\Omega-C$, because $\int_{C} G(x, y) \mu_{K}^{2}(d y)$ is harmonic in $\Omega-C$. Furthermore $v(x)$ is superharmonic in $Q$, because it holds that

$$
\begin{aligned}
& v(x)=\int_{Q} G(x, y) \mu_{K}^{1}(d y)-\int_{\Omega} G(x, y) \mu_{K}^{2}(d y)+\int_{\Omega-C} G(x, y) \mu_{K}^{2}(d y) \\
& =-\left(\int_{\Omega} G(x, y) \mu_{K}^{1}(d y)-\int_{Q} G(x, y) \mu_{K}^{1}(d y)\right)+\int_{\Omega-C} G(x, y) \mu_{K}^{2}(d y)
\end{aligned}
$$

and the first term of the right-hand side is harmonic in $Q$. Therefore $v(x)$ is superharmonic in $\Omega$. So we have $E_{x} v\left(x_{\tau_{\sigma_{n}}}\right) \leqq v(x)$ for each compact set $G_{n}$ such that $G_{n} \uparrow \Omega$. Noting Lemma 3 , we have $v(x) \geqq 0$. As $C$ is arbitrary, it holds

$$
\int_{Q} G(x, y) \mu_{K}^{1}(d y) \geqq \int_{Q} G(x, y) \mu_{K}^{2}(d y) .
$$

We can also prove that

$$
\int_{Q} G(x, y) \mu_{K}^{2}(d y) \geqq \int_{Q} G(x, y) \mu_{K}^{1}(d y) .
$$

The case where b) is satisfied, will be discussed at the next section.

\$3. Construction of Feller process having the Green function with singularity

Our aim of this section is to show the following Theorem.

THEOREM 2. For a given kernel $G(x, y)$ with singularity $\varphi \in \Phi$ which satisfies the complete maximum principle, there exists a Feller process whose Green function is $G(x, y)$.

Proof. This Theorem is an extension of Theorem 1.1 in [6]. The proof carried out with only obvious modifications of Theorem 1. 1. if we note the following: Let $Q$ (resp. $Q^{\prime}$ ) be a ball with radius $r$ (resp. $2 r$ ) which centers at $x_{0}$. Then we have 
a) $\quad \sup _{x \in Q} \int_{Q} \varphi(|x-y|) d y=\int_{Q} \varphi\left(\left|x_{0}-y\right|\right) d y$,
b) $\quad 1 \leqq \frac{\sup _{x \in Q^{\prime}} \int_{Q^{\prime}} \varphi(|x-y|) d y}{\int_{Q} \varphi\left(\left|x_{0}-y\right|\right) d y} \leqq 1+2^{d}$,

for each function $\varphi \in \Phi$, if $Q^{\prime}$ is sufficiently small. The equality $a$ ) can be shown by a simple culculation. The inequality $b$ ) is proved as follows;

$$
\begin{aligned}
& \stackrel{\sup _{x \in Q^{\prime}} \int_{Q^{\prime}} \varphi(|x-y|) d y}{\int_{Q} \varphi\left(\left|x_{0}-y\right|\right) d y}=\frac{\int_{Q^{\prime}} \varphi\left(\left|x_{0}-y\right|\right) d y}{\int_{Q} \varphi\left(\left|x_{0}-y\right|\right) d y} \\
& =\frac{\int_{Q^{\prime}-Q} \varphi\left(\left|x_{0}-y\right|\right) d y+\int_{Q} \varphi\left(\left|x_{0}-y\right|\right) d y}{\int_{Q} \varphi\left(\left|x_{0}-y\right|\right) d y} \\
& \leqq 1+\frac{\varphi(r)\left\{V\left(Q^{\prime}\right)-V(Q)\right\}}{\varphi(r) V(Q)}=1+2^{d},
\end{aligned}
$$

where $V(Q)$ denotes a volume of $Q$.

Remark 1. An important example of a Feller process having Green function $G(x, y)$ is a process corresponding to the kernel $G(x, y)=\varphi(|x-y|)$, where $\varphi \in \Phi_{M}^{1}$. We shall give an example of a function $\varphi(t) \in \Phi_{M}^{1}$ in $R^{3}$ whose singularity is $t^{3-\alpha} \log \frac{1}{t}$ in $\$ 8$.

Corollary. Let $X=\left(x_{t}, \zeta, M_{t}, P_{x}\right)$ be a Feller process which has quasisymmetric Green function $G(x, y)$ with singularity $\varphi \in \Phi$. Then there exists a Feller process $\hat{X}=\left(\hat{x}_{t}, \hat{\zeta}, \hat{M}_{t}, \hat{P}_{x}\right)$ which has Green function $\hat{G}(x, y)(=G(y, x))$ such that

$$
\int_{\Omega} f(x) G_{\alpha} g(x) d x=\int_{\Omega} g(x) \hat{G}_{\alpha} f(x) d x
$$

holds for each $f, g \in B_{K}$. and $\alpha \geqq 0$.

We call the process $\hat{X}$ the dual process of $X$. Our processes $X$ and $\hat{X}$ are in the relation of duality in Meyer's sense. (See [12].) Hence we have the following

Lemma 1. (Hunt). Let $X$ and $\hat{X}$ be Feller processes in Corollary. Then we have 


$$
\int_{\Omega} G(z, y) P_{x}\left(x \sigma_{E} \in d z\right)=\int_{\Omega} G(x, z) \hat{P}_{y}\left(\hat{x}_{\sigma_{E}} \in d z\right)
$$

for each nearly analytic set $E$.

Proof. This is shown by G.A. Hunt [4] under Hunt's hypothesis (F) and (G). Further, P.A. Meyer [12] showed it under a little weaker conditions. Our lemma follows directly from P.A. Meyer's [12]. (See also [7].)

\$4. Capacitary measures for a Feller process having the quasisymmetric Green function

Throughout this section we always consider a Feller process $X=\left(x_{t}\right.$, $\left.\zeta, M_{t}, P_{x}\right)$ having the quasi-symmetric Green function $G(x, y)$ with singularity $\varphi$ and its dual process $\hat{X}=\left(\hat{x}_{t}, \zeta, \hat{M}_{t}, \hat{P}_{x}\right)$ without special mentioning. We study some properties about capacitary measures which are analogous to those of G.A. Hunt [4]. We first note the following

Lemma 1. The capacitary measure is uniquely determined.

Proof. Our case can be treated in the general setting of $\mathrm{H}$. Kunita and T. Watanabe [10] (See also [7]). Hence the measure is uniquely determined by its potential (See Prop. 7. 11 in H. Kunita and T. Watanabe $[10])$.

Let $E$ be a nearly analytic set. We say that a point $x$ is a regular point of $E$ for $X$ (resp. $\hat{X})$, if $P_{x}\left(\sigma_{E}=0\right)=1 \quad$ (resp. $\hat{P}_{x}\left(\hat{\sigma}_{E}=0\right)=1$ ) and an irregular point of $E$ for $X$ (resp. $\hat{X})$, if $P_{x}\left(\sigma_{E}=0\right)=0$ (resp. $\hat{P}_{x}\left(\hat{\sigma}_{E}=0\right)=0$ ). In the following a regular (resp. irregular) point of $E$ for $X$ is called simply a regular (resp. irregular) point of $E$ and a regular (resp. irregular) point of $E$ for $\hat{X}$ is called simply a co-regular (resp. co-irregular) point of $E$. We denote by $E^{r e g}, E^{c o-r e g}, E^{i r r e g}$ and $E^{c o-i r r e g}$ the set of all the points which are regular, co-regular, irregular and co-irregular of $E$ respectively. Nextly we prepare some elementary properties about capacitary measures.

Lemma 2. For each nearly analytic set $E$ with compact closure and for each $\varepsilon>0$, there exists a nearly analytic set $F$ such that $E \subset F^{r e g}$ and $P_{\mu}\left(\sigma_{E}<+\infty\right)+$ $\varepsilon \geqq P_{\mu}\left(\sigma_{F}<+\infty\right)$ for each measure $\mu$ with $\mu\left(E \cap E^{i r r}\right)=0$, where $F$ may depends on $\mu$.

Remark. Lemma 2 is also shown for the Feller process which has a locally integrable Green function in the sense of P.A. Meyer [11], (See the proof $C$ ) of Theorem 3.5 in [11]). 
L.emma 3. For each nearly analytic set $E$ with compact closure, the capacitary measure $\mu_{E}(d y)$ is concentrated on $E \cup E^{c o-r e g}$.

Proof. The proof is same as that of G.A.Hunt [4], Prop. 18. 4. We omit it here.

Remark 1. Using Lemma 3, we can easily see that $\mu_{E}(\bar{E})=\hat{\mu}_{E}(\bar{E})$ on the same way as in G.A.Hunt [4], p. 175.

Now we shall show two properties about capacitary measures for the process which satisfies Hunt's condition $(H)$ :

$(H)$;

$$
\text { if } F \text { is compact and } \mu_{F}(F)>0 \text {, then some }
$$

point of $F$ is a regular point of $F$.

Under the condition $(H)$ it holds that $\mu_{K}\left(K \cap K^{c o-i r r}\right)=0$ for each nearly analytic set $K$ with compact closure. (note that $K \cap K^{\text {co-irr }}$ is $\mu$ measurable for any bounded measure $\mu_{\text {. }}$ ) Indeed, if we assume $\mu_{K}\left(K \cap K^{\text {co-irr }},{ }^{2}\right)>0$, there exists a compact set $Q \subset K \cap K^{\text {co-irr }}$ such that $\mu_{K}(Q)>0$, which means $\mu_{Q}(Q) \geqq \mu_{K}(Q)>0$.

Then some point of $Q$ is a co-regular point of $Q$ (of course it is a coregular point of $K$ ), which is a contradiction.

Lemma 4. Under the condition $(H)$, for each compact set $K$, there exists a sequence of nearly analytic sets $\left\{Q_{n}\right\}$ such that $Q_{n} \downarrow K, Q_{n}^{\text {co-reg }} \supset K$ and it holds

$$
\mu_{Q_{n}}\left(\bar{Q}_{n}\right) \downarrow \mu_{K}(K) .
$$

Proof. Let $Q$ be a bounded open set which includes $K$. Then, for the capacitary measure $\mu_{Q}(d y)$, we can choose a sequence of nearly analytic sets $\left\{Q_{n}\right\}$ with $Q_{n} \downarrow K, Q \supset \bar{Q}_{n}, Q_{n}^{\text {co-reg }} \supset K$ such that

$$
\hat{P}_{\mu_{Q}}\left(\hat{\sigma}_{Q_{n}}<+\infty\right) \downarrow \hat{P}_{\mu_{Q}}\left(\hat{\sigma}_{K}<+\infty\right)
$$

by Lemma 2 because $\mu_{Q}\left(K \cap K^{c o-i r r}\right) \leqq \mu_{K}\left(K \cap K^{c o-i r r}\right)=0$. On the other hand we have

$$
\begin{aligned}
\mu_{Q_{n}}\left(\bar{Q}_{n}\right) & =\hat{\mu}_{Q_{n}}\left(\bar{Q}_{n}\right)=\int_{\bar{Q}_{n}} P_{x}\left(\sigma_{Q}<+\infty\right) \hat{\mu}_{Q_{n}}(d x)=\int_{\bar{Q}_{n}} \int_{\bar{Q}} G(x, y) \mu_{Q}(d y) \hat{\mu}_{Q_{n}}(d x) \\
& =\int_{\bar{Q}} \hat{P}_{y}\left(\hat{\sigma}_{Q_{n}}<+\infty\right) \mu_{Q}(d y)
\end{aligned}
$$


and

$$
\mu_{K}(K)=\int_{\bar{Q}} \hat{P}_{y}\left(\hat{\sigma}_{K}<+\infty\right) \mu_{Q}(d y)
$$

Lemma 5. Under the condition $(H)$, for each compact set $K$, we have

$$
\mu_{K}(K)=\sup _{\nu \in M_{K}} \nu(K)
$$

where $M_{K}=\{$ measure $\nu, G \nu \leqq 1, S(\nu)(=$ support of $\nu) \subset K\}$.

Proof. For a sequence of $\left\{Q_{n}\right\}$ in Lemma 3 and $\nu \in M_{K}$, it holds

$$
\begin{aligned}
\mu_{Q_{n}}\left(\bar{Q}_{n}\right) & =\hat{\mu}_{Q_{n}}\left(\bar{Q}_{n}\right) \geqq \int_{\bar{Q}_{n}} G \nu(x) \hat{\mu}_{Q_{n}}(d x) \\
& =\int_{K} \hat{P}_{y}\left(\hat{\sigma}_{Q_{n}}<+\infty\right) \nu(d y)=\nu(K) .
\end{aligned}
$$

Hence, noting Lemma 3 , we have $\mu_{K}(K) \geqq \sup _{\nu \in M_{K}} \nu(K)$. Since it is clear that $\mu_{K}(K) \leqq \sup _{\nu \in M_{K}} \nu(K)$, we finish the proof.

It is unknown whether Hunt's condition $(H)$ holds for general Feller process having the quasi-symmetric Green function with singularity $\varphi \in \Phi$. But in special case we can show that $(H)$ holds by proving the continuity principle (Hunt's condition (I), [4]).

Lemma 6. Suppose that the function $\varphi$ belongs to the class $\Phi_{1}$. Then the condition $(H)$ holds. (Hence the condition $(B)$ holds).

Proof. i) We shall first show the following. Let $\mu$ be a bounded measure with compact support $S(\mu)$, such that $G \mu$ is bounded, then, for each $\mu$-measurable subset $Q$ of $S(\mu)$ which is contained in some ball $\tilde{Q}$ with radius $\frac{r}{4}(r$ denotes a strictly positive constant such that $\varphi(t)$ is defined on $(0, r]$ and $t^{p} \varphi(t)$ is monotone decreasing), it holds that

$$
\int_{Q} G(x, y) \mu(d y) \leqq L \cdot \sup _{x^{\prime} \in \bar{Q}} \int_{Q} G\left(x^{\prime}, y\right) \mu(d y), \quad \forall x \in \Omega,
$$

where $L$ is a positive constant independent of $Q$ (dependent on $\tilde{Q}$ and $\mu$ ). Let $V$ be a $\frac{r}{4}$-neighborhood of $\bar{Q}$, then, for each $x_{0} \in \bar{V}-\bar{Q}$, there exists a point $y_{0} \in \bar{Q}$ such that $\inf _{y \in Q}\left|x_{0}-y\right|=\left|x_{0}-y_{0}\right|$ and $2^{p} \varphi\left(\left|y_{0}-y\right|\right) \geqq \varphi\left(\left|x_{0}-y\right|\right)$, because $\left|y_{0}-y\right| \leqq\left|y_{0}-x_{0}\right|+\left|x_{0}-y\right| \leqq 2\left|x_{0}-y\right|, y \in \bar{Q}$. Hence we have 


$$
\int_{Q} G\left(x_{0}, y\right) \mu(d y) \leqq \frac{\sup _{(x, y) \in \overline{\tilde{Q}} \times \bar{Q}} C(x, y)}{\inf _{(x, y) \in \tilde{\tilde{Q}} \times \overline{\tilde{Q}}} C(x, y)} \cdot 2^{p} \cdot \int_{Q} G\left(y_{0}, y\right) \mu(d y)
$$

For each point $x \in \bar{V}^{c}$ we have, noting $\bar{Q} \subset V^{\text {co-reg }}$,

$$
\int_{Q} G(x, y) \mu(d y)=E_{x} \int_{Q} G\left(x_{\tau_{v}}, y\right) \mu(d y) \leqq \sup _{x \in \bar{V}} \int_{Q} G(x, y) \mu(d y)
$$

Hence (4. 1) is proved.

ii). By using the inequality (4.1) we shall show the following. Let $\mu$ be a measure mentioned in i). If $G \mu$ is continuous on $S(\mu)$, it is continuous everywhere. Since $G \mu$ is continuous on $S(\mu)^{c}$, we have only to show that $G \mu$ is continuous at $\partial S(\mu)$. Let $x_{0} \in \partial S(\mu)$ and $\left\{Q_{n}\left(x_{0}\right)\right\}$ be a sequence of open balls such that $Q_{n}\left(x_{0}\right) \downarrow x_{0}$, then we have

$$
\int_{s(\mu)-Q_{n}\left(x_{0}\right)} G(x, y) \mu(d y) \uparrow \int_{s(\mu)} G(x, y) \mu(d y)
$$

and the both sides are continuous on $S(\mu)$, because

$$
G \mu(x)=\int_{s(\mu)-Q_{n}\left(x_{0}\right)} G(x, y) \mu(d y)+\int_{Q_{n}\left(x_{0}\right) \cap S(\mu)} G(x, y) \mu(d y)
$$

and $G \mu$ is continuous on $S(\mu)$ and $\int_{Q_{n}\left(x_{0}\right)} G(x, y) \mu(d y)$ is lower semi-continuous. Hence, by Dini's theorem, its convergence is uniform on $S(\mu)$, which means

$$
\int_{Q_{n}\left(x_{0}\right) \cap S(\mu)} G(x, y) \mu(d y) \downarrow 0 \text {, uniformly on } S(\mu) \text {. }
$$

Hence the proof is completed. (The proof of ii) is due to S. Watanabe [15].)

iii). Using the result of ii) we can show that the following condition $\left(J^{\prime}\right)$ weaker than Hunt's condition $(J)$ holds for the potential in (i) on the same way as in G.A. Hunt [4], p. 195.

$\left(J^{\prime}\right)$; For each $\varepsilon>0$ there exists an open set $Q$ such that $\mu_{Q}(\bar{Q})<\varepsilon$ and the restriction of $G \mu$ to $\Omega-Q$ is continuous.

iv). From $\left(J^{\prime}\right)$ we can also show that the following condition $\left(K^{\prime}\right)$ weaker than Hunt's condition $(K)$ holds on the same way as in G.A. Hunt [4], p. 197. 
Let $G \mu$ be a potential mentioned in $\left(J^{\prime}\right)$.

$\left(K^{\prime}\right)$; Then, $G \mu\left(x_{t}\right)$ is continuous whenever $x(t, w)$ is continuous for almost all $w$ with respect to $P_{f}$-measure, where $P_{f}(\cdot)=\int_{\Omega} P_{x}(\cdot) f(x) d x$ and $f(x)$ is a non-negative bounded measurable function with compact support.

v). That $\left(K^{\prime}\right)$ implies $(H)$ can be established by the same way as in the proof $(a)$ of Theorem 6. 5 in P.A.Meyer [13]. (See also S.Watanabe [15]), p. 34).

\section{§5. Maximum principle and the singularity}

This section is devoted to the proof of Theorem 3. We prove it by the contradiction. Without special mentioning the process $X=\left(x_{t}, \zeta, M_{t}, P_{x}\right)$ we concern is a Feller process having the quasi-symmetric Green function $G(x, y)$ with singularity $\varphi \in \Phi$ and the sets we concern are assumed to be sufficiently small.

Let $\varepsilon_{\partial Q}(d y)$ be the uniform measure on the surface $\partial Q$ of a ball $Q$ whose total mass is 1 .

If we set

$$
\begin{aligned}
& L=\frac{1}{\sqrt{\pi}} \frac{\Gamma\left(\frac{d}{2}\right)}{\Gamma\left(\frac{d-1}{2}\right)} \int_{0}^{\pi} \varphi\left(2 r \sin \frac{\theta}{2}\right) \sin ^{d-2} \theta d \theta, \\
& \left(=\int \varphi(|x-y|) \varepsilon_{\partial Q}(d y) ; x \in \partial Q\right),
\end{aligned}
$$

where $r$ is the radius $Q$, then we have the following

LEMMA 1. If $L$ is finite, it holds

$$
\frac{1}{m_{1}} L^{-1} \geqq \mu_{\partial Q}(\partial Q) \geqq \frac{m_{1}}{m_{2}} \cdot \frac{1}{m_{2}} \cdot L^{-1},
$$

where $m_{1}=\inf _{(x, y) \in O_{x_{0}} \times O_{x_{0}}} C(x, y), m_{2}=\sup _{(x, y) \in O_{x_{0}} \times O_{x_{0}}} C(x, y) . \quad$ (Here a ball $Q$ centering at $x_{0}$ is assumed to be in some $O_{x_{0}}$.)

Proof. The first inequality is proved as follows.

$$
\begin{aligned}
\varepsilon_{\partial Q}(\partial Q) & \geqq \int_{\Omega} P_{x}\left(\sigma_{Q}<+\infty\right) \varepsilon_{\partial Q}(d x) \\
& \geqq m_{1} \iint_{\Omega \times \Omega} \varphi(|x-y|) \mu_{\partial Q}(d y) \varepsilon_{\partial Q}(d x) \\
& =m_{1} L \cdot \mu_{\partial Q}(\partial Q) .
\end{aligned}
$$


We shall next show the second inequality. By the remark of Lemma 2 of $\S 4$, for any $\varepsilon>0$ there exists a nearly analytic set $E$ such that $E^{r e g} \supset \partial Q$,

$$
P_{x_{0}}\left(\sigma_{E}<+\infty\right) \leqq P_{x_{0}}\left(\sigma_{\partial Q}<+\infty\right)+\varepsilon \text {. }
$$

Since we may assume that

$$
\begin{aligned}
& \varphi(r)-\varepsilon \leqq \inf _{y \in \bar{E}} \varphi\left(\left|x_{0}-y\right|\right) \\
& \sup _{x \in \bar{E}} \int_{\Omega} \varphi(|x-y|) \varepsilon_{\partial Q}(d y) \leqq L(1+\varepsilon),
\end{aligned}
$$

the following inequality holds

$$
\begin{aligned}
\mu_{E}(\bar{E}) & \geqq \int_{\Omega}\left\{\frac{1}{L} \int_{\Omega} \varphi(|x-y|) \varepsilon_{\partial Q}(d y)-\varepsilon\right\} \mu_{E}(d x) \\
& \geqq \frac{1}{m_{2} \cdot L} \int_{\Omega} P_{x}\left(\sigma_{E}<+\infty\right) \varepsilon_{\partial Q}(d x)-\varepsilon \mu_{E}(\bar{E}) \\
& =\frac{\varepsilon_{\partial Q}(\partial Q)}{m_{2} \cdot L}-\varepsilon \mu_{E}(\bar{E}) .
\end{aligned}
$$

Noting that

$$
m_{2} \cdot \varphi(r) \cdot \mu_{\partial Q}(\partial Q)+\varepsilon \geqq m_{1} \cdot\{\varphi(r)-\varepsilon\} \mu_{E}(\bar{E}),
$$

we have

$$
\mu_{\partial Q}(\partial Q) \geqq \frac{m_{1}}{m_{2}} \cdot \frac{1}{m_{2} \cdot L}\left\{1-\frac{\varepsilon}{\varphi(r)}\right\} \frac{1}{1+\varepsilon}-\frac{\varepsilon}{m_{2} \cdot \varphi(r)} .
$$

Since $\varepsilon$ is arbitrary, we finally obtain the second inequality.

Proof of Theorem 3. Suppose the kernel $G(x, y)$ of Theorem 3 satisfies the complete maximum principle, then there exists a Feller process whose Green function is $G(x, y)$ by Theorem 2. Therefore it holds by Lemma 1 in $\S 4$

$$
\begin{aligned}
& 1 \geqq P_{x_{0}}\left(\sigma_{\partial Q}<+\infty\right)=\int_{\Omega} G\left(x_{0}, y\right) \mu_{\partial Q}(d y) \\
& \geqq m_{1} \int_{\Omega} \varphi\left(\left|x_{0}-y\right|\right) \mu_{\partial Q}(d y)=m_{1} \cdot \varphi(r) \cdot \mu_{\partial Q}(\partial Q)
\end{aligned}
$$

for each ball $Q \subset O_{x_{0}}$ with radius $r$ which centers at $x_{0}$. Hence by Lemma 1 we have

$$
1 \geqq\left(\frac{m_{1}}{m_{2}}\right)^{2} \varphi(r) \cdot \frac{1}{L}
$$


Noting that $C(x, y)$ is continuous in $(x, y)$, we can choose a sufficiently small ball $Q$ such that $\left(\frac{m_{1}}{m_{2}}\right)^{2}>\frac{1}{M_{1}} \Gamma\left(\frac{d}{2}\right) \Gamma\left(\frac{d-1}{2}\right)^{-1}$, where $M_{1}$ is a constant mentioned in Theorem 3. Then the above inequality implies

$$
M_{1} \int_{0}^{\pi} \varphi\left(2 r \sin \frac{\theta}{2}\right) \sin ^{d-2} \theta d \theta>\sqrt{\pi} \varphi(r),
$$

which is equivalent to the inequality in Theorem 3.

Remark 1. There exists a case in which Theorem 3 holds for $M_{1}=\Gamma\left(\frac{d}{2}\right) \Gamma\left(\frac{d-1}{2}\right)^{-1}$. In fact, if $G(x, y)$ is a function of the distance between $x$ and $y$ and if $\varphi(|x-y|)=G(x, y)$ satisfies the inverse inequality of Theorem 3 with $M_{1}=\Gamma\left(\frac{d}{2}\right) \Gamma\left(\frac{d-1}{2}\right)^{-1}$, then $G(x, y)$ does not satisfy the complete maximum principle.

Corollary. When $d>\alpha>2$, a kernel with singularity $t^{\alpha-d}$ is not a quasi-symmetric Green function under the continuity assumption of $C(x, y)\left(=\frac{G(x, y)}{\varphi(|x-y|)}\right)$.

Proof. We may apply Theorem 3 by taking $\varphi(t)=t^{\alpha-\alpha}$, but the culculation of $\Gamma$-function is not easy. So we prove this as follows. Noting that $\Delta \cdot t^{\alpha-d}<0$ for $2<\alpha<d$, where $\Delta$ is the Laplacian, we have $\int_{\Omega} \varphi\left(\left|x_{0}-y\right|\right) \varepsilon_{\partial Q}(d y)>L$. On the other hand, we can show by an elementary culculation that $L^{-1} \int_{\Omega} \varphi\left(\left|x_{0}-y\right|\right) \varepsilon_{\partial Q}(d y)$ is a constant independent of $Q$. Hence the inequality in Theorem 3 does not holds.

\section{\$6. Continuity of sample paths and the singularity}

In this section we shall prove Theorem 4. Let us first note the following lemma.

Lemma 1. Let $X=\left(x_{t}, \zeta, M_{t}, P_{x}\right)$ be a Feller process on $\Omega$ such that each point is not a trap. If almost all paths starting at $x_{0}$ are continuous, for each positive number $\varepsilon$ there exists a ball $\tilde{Q}$ such that $P_{x_{0}}\left(\sigma_{\partial \hat{Q}}<+\infty\right)>1-\varepsilon$ holds for each ball $Q \subset \tilde{Q}$.

Proof. Now if we choose a sequence of open balls $\left\{Q_{n}\right\}$ with radius $n^{-1}$ which centers at $x_{0}$, almost every path meets some $\partial Q_{n}\left(\partial Q_{n}\right.$ depends on the path $w)$ by the continuity of the path, because $P_{x_{0}}(\zeta>0)=1$. Noting that the sequence of events $\left\{\sigma_{\partial Q_{n}}<+\infty\right\}$ is increasing by the con- 
tinuity of the path, if there exists some constant $\varepsilon>0$ such that $P_{x_{0}}\left(\sigma_{\partial Q_{n}}\right.$ $<+\infty)<1-\varepsilon$ for each $n$, then it holds that $P_{x_{0}}\left(\bigcup_{n}\left(\sigma_{\partial Q_{n}}<+\infty\right)\right) \leqq 1-\varepsilon$, which contradicts the above statement.

Proof of Theorem 4. We use the same notations as those of the proofs in \$4. If the inequality about $\varphi(t)$ in Theorem 4 holds, we have

$$
\varphi(r) \cdot L^{-1}<M_{2} \cdot \Gamma\left(\frac{d}{2}\right)^{-1} \cdot \Gamma\left(\frac{d-1}{2}\right)
$$

Hence it holds that

$$
\frac{m_{1}}{m_{2}} \int_{\Omega} G\left(x_{0}, y\right) \mu_{\partial Q}(d y) \leqq \varphi(r) \cdot m_{1} \cdot \mu_{\partial Q}(\partial Q) \leqq \varphi(r) \cdot L^{-1}<M_{2} \cdot \Gamma\left(\frac{d}{2}\right)^{-1} \Gamma\left(\frac{d-1}{2}\right) .
$$

Using the continuity assumption of $C(x, y)$ we have

$$
\int_{\Omega} G\left(x_{0}, y\right) \mu_{\partial Q}(d y)<M_{2}^{\prime}<1
$$

for each sufficiently small ball $Q$ centering at $x_{0}$, which implies that the process is not a continuous process by Lemma 1 .

Proposition. Let $X$ be a Feller process which has the Green function with singularity $\varphi \in \Phi$. If the process $X$ satisfies Hunt's condition $(H)$ and $\frac{t^{1-d}}{\varphi(t)}$ is monotone increasing, then $X$ is not a continuous process even if $L$ in Lemma 1 of $\$ 5$ is infinite.

Proof. It is sufficient to show that $\mu_{\partial Q}(\partial Q)=0$. If we assume that there exists a capacitary measure $\mu_{\partial Q}(d y)$ such that $\mu_{\partial Q}(\partial Q)>0$, some points are regular points of $\partial Q$ for $X$ by the condition $(H)$. Using the result of Theorem 5 which will be shown in the next section, such a point is a regular point of $\partial Q$ for the symmetric stable process $X^{s}$ with exponent 1 . But $\partial Q^{r e g}$ is empty for $X^{s}$. Thus we have proved the theorem.

Corollary. Let $X$ be a Feller process having the Green function $G(x, y)$ with singularity $t^{\alpha-a}$. Under the continuity assumptions on $C(x, y)$, if $0<\alpha<2$, $X$ is not a continuous process.

Proof. Since $X$ satisfies the condition $(H)$ in case $0<\alpha \leqq 1$ (for the proof see Proposition in \$7), $X$ is not a continuous process by the above proposition because $\frac{t^{1-d}}{t^{\alpha-d}}=t^{1-\alpha} \uparrow$ if $\alpha \leqq 1$. Let us set $\varphi(t)=t^{\alpha-d}$, then for the case $2>\alpha>1$ there exists a constant $K<1$ such that 


$$
\int_{\Omega} \varphi\left(\left|x_{0}-y\right|\right) \varepsilon_{\partial Q}(d y)<K \cdot L
$$

Indeed, we have only to note that $\Delta \cdot t^{\alpha-d}>0$ for $0<\alpha<2$ and that $\int_{\Omega} \varphi(|x-y|) \varepsilon_{\partial Q}(d y)=L$ on $\partial Q, L^{-1} \int_{\Omega} \varphi\left(\left|x_{0}-y\right|\right) \varepsilon_{\partial Q}(d y)$ is constant independently of a choice of a ball $Q$ centering at $x_{0}$. Hence by the continuity of $C(x, y)\left(=\frac{G(x, y)}{\varphi(|x-y|)}\right)$ we see that the inequality about $\varphi$ in Theorem 4 holds for $\varphi(t)=t^{\alpha-d}$ in case $1<\alpha<2$.

Remark. It is well known that to the Riesz kernel with exponent 2Newtonian kernel-, there corresponds Brownian motion. But to general kernel with singularity $t^{2-d}$ a continuous process does not always correspond. We shall give such an example in $R^{3}$.

Let us set

$$
G(x, y)=\mathscr{F}^{-1}\left(\frac{1}{|z|^{2}+|z|^{\beta}}\right)(|x-y|), x, y \in R^{3},
$$

where $\mathscr{F}^{-1}$ denotes the inverse Fourier transform and $1<\beta<2$. Suppose for a moment that $G(x, y)$ is a kernel in $R^{3}$ with singularity $t^{2-d}, d=3$, which satisfies the complete maximum principle. (The proof will be given in the next paragraph.) Then the Feller process whose Green function is the above $G(x, y)$ is not continuous. Indeed, since $|x|^{2}+|x|^{\beta}$ is a negative definite function there exists a generalized Laplacian $A$ such that

$$
\mathscr{F}(A)=-\left(|x|^{2}+|x|^{\beta}\right) d x,
$$

and further we can see that

$$
\begin{gathered}
A * u(x)=C_{1} \cdot \Delta \cdot u(x)+C_{2} \cdot \int_{R^{3}-(0)}\left\{u(y+x)-u(x)-\sum_{i=1}^{3} \frac{\partial}{\partial x_{i}} u(x)\left(y_{i}-x_{i}\right) \frac{1}{1+|y|^{2}}\right\} \\
f(y) d y, f(y) d y \text {; Lévy messure for } \beta \text {-stable process, }
\end{gathered}
$$

where $\Delta$ is the Laplacian and $C_{1}, C_{2}$ are strictly positive constants suitably chosen. As $A$ is not a local operator, the process corresponding to $G(x, y)$ is not a continuous process.

It remains to show that the kernel $G(x, y)$ has singularity $\varphi \in \Phi$ and satisfies the complete maximum principle. Since it holds that

$$
G(x, y)=\lim _{A \rightarrow+\infty} \frac{2}{|x-y|} \int_{0}^{A} \frac{\sin 2 \pi r}{r+|x-y|^{2-\beta} r^{\beta-1}} d r
$$


we can see that by the second mean value theorem

$$
G(x, y)=\frac{C(|x-y|)}{|x-y|},
$$

where $C(|x|)$ is bounded in $R^{3}$. The continuity of $G(x, y)$ except at the diagonal can be proved easily. By Lemma 1 in the latter section 8, $G(x, y)$ is positive almost everywhere and so positive everywhere. Furthermore, by Lemma 2 in $\S 8, G(x, y)$ satisfies the complete maximum principle.

\section{\$7. Regular points and the singularity}

The results in this section are improvements of our previous one [6]. We shall first show that the Wiener test holds in the following sense.

Proposition. (Wiener test). Let $X=\left(x_{t}, \zeta, M_{t}, P_{x}\right)$ be a Feller process satisfying the condition $B$ on $\Omega$ which has the Green function with singularity $\varphi$. Suppose $\varphi \in \Phi_{1}$. Then a point $x_{0}$ is a regular point of a nearly analytic set $B$ for $X$, if and only if

$$
\sum_{k=1}^{+\infty} \varphi\left(\frac{1}{2^{k}}\right) \mu_{B_{k}}\left(\bar{B}_{k}\right)=+\infty
$$

where $B_{k}=\left\{x ; \frac{1}{2^{k+1}} \leqq\left|x-x_{0}\right| \leqq \frac{1}{2^{k}}\right\} \cap B$ and $\mu_{B_{k}}(d y)$ is the capacitary measure of $B_{k}$.

Proof. This proposition can be proved by the same method as that of Theorem 4. 1 in [6], if we only note that $1 \leqq \frac{\varphi\left(\frac{t}{2}\right)}{\varphi(t)} \leqq 2^{p}$. We omit the proof here. We also note that

$$
\sum_{k=1}^{+\infty} \varphi\left(\frac{1}{2^{k}}\right) \mu_{B_{k}}\left(\widetilde{B}_{k}\right)=+\infty
$$

if and only if

$$
\sum_{k=1}^{+\infty} P_{x_{0}}\left(\sigma_{B_{k}}<+\infty\right)=+\infty .
$$

Proof of i) of Theorem 5. We shall first prove the Theorem for a compact set $B$ in general case. Let the sequence $\left\{B_{k}\right\}$ be the sequence of sets defined in the previous proposition and $\mu_{B_{k}}^{1}(d y)$ and $\mu_{B_{k}}^{2}(d y)$ be capacitary measures of $B_{k}$ for $X^{1}$ and $X^{2}$ respectively. Then we can choose a sequence of nearly analytic set $\left\{G_{k}\right\}$ such that $G_{k}^{r e g} \supset B_{k}$, $\varphi_{2}$ (diameter of $\left.B_{k}\right) \times \varphi_{1}$ (diameter of $\left.B_{k}\right)^{-1} \leqq 2 \varphi_{2}$ (diameter of $\left.G_{k}\right) \times \varphi_{1}\left(\text { diameter of } G_{k}\right)^{-1}$ and such that 
$P_{x_{0}}^{1}\left(\sigma_{G_{k}}<+\infty\right) \leqq P_{x_{0}}^{1}\left(\sigma_{B_{k}}<+\infty\right)+\frac{1}{2^{k}}\left\{\varphi\left(\frac{1}{2^{\bar{k}}}\right)\right\}^{-1}$ (note that $B_{k}$ is compact). Hence, for $B_{k}$ with sufficiently large suffix $k$, we have

$$
\begin{aligned}
\mu_{G_{k}}^{1}\left(\bar{G}_{k}\right) & \geqq \int_{\Omega} P_{x}^{2}\left(\sigma_{B_{k}}<+\infty\right) \mu_{G_{k}}^{1}(d x) \\
& =\iint_{\Omega \times \Omega} G^{2}(x, y) \mu_{B_{k}}^{2}(d y) \mu_{G_{k}}^{1}(d x) \\
& \geqq C_{1} \iint_{\Omega \times \Omega} \frac{\varphi_{2}(|x-y|)}{\varphi_{1}(|x-y|)} G^{1}(y, x) \mu_{G_{k}}^{1}(d x) \mu_{B_{k}}^{2}(d y) \\
& \geqq \frac{C_{2}}{2} \iint_{\Omega \times \Omega} \frac{\varphi_{2}\left(\text { diameter of } B_{k}\right)}{\varphi_{1}\left(\text { diameter of } B_{k}\right)} P_{y}^{1}\left(\sigma_{G_{k}}<+\infty\right) \mu_{B_{k}}^{2}(d y),
\end{aligned}
$$

where $C_{1}$ and $C_{2}$ are strictly positive constants which can be chosen independently of $k_{\text {. }} \quad$ Note that

$$
\frac{\varphi_{2}\left(\text { diameter of } B_{k}\right)}{\varphi_{1}\left(\text { diameter of } B_{k}\right)} \geqq \varphi_{2}\left(\frac{2}{2^{k}}\right) \varphi_{1}\left(-\frac{2}{2^{k}}\right)^{-1} \geqq 2^{-p} \varphi_{2}\left(\frac{1}{2^{k}}\right) \cdot \varphi_{1}\left(\frac{1}{2^{\bar{k}}}\right)^{-1} \text {, }
$$

where $p_{2}$ is a strictly positive constant that $t^{p_{2}} \varphi_{2}(t)$ is monotone increasing and $\lim _{t \rightarrow 0} t^{p_{2}} \varphi_{2}(t)=0$. Then it holds that

$$
\mu_{G_{k}}^{1}\left(\bar{G}_{k}\right) \geqq \frac{C_{2}}{2^{p_{2}+1}} \frac{\varphi_{2}\left(2^{-k}\right)}{\varphi_{1}\left(2^{-k}\right)} \mu_{B_{k}}^{2}\left(\bar{B}_{k}\right) .
$$

Finally we have

$$
\sum_{k=1}^{+\infty} \varphi_{1}\left(2^{-k}\right) \mu_{G_{k}}^{1}\left(\bar{G}_{k}\right) \geqq C_{3} \sum_{k=1}^{+\infty} \varphi_{2}\left(2^{-k}\right) \mu_{B_{k}}^{2}\left(\bar{B}_{k}\right),
$$

where $C_{3}$ is a strictly positive constant. Since $\sum_{k=1}^{+\infty} \varphi_{1}\left(-\frac{1}{2^{k}}\right) \mu_{G_{k}}^{1}\left(\bar{G}_{k}\right)$ and $\sum_{k=1}^{+\infty} P_{x_{0}}\left(\sigma_{G_{k}}<+\infty\right)$ diverges simultaneously, the proof is complete, if we note that $\sum_{k=1}^{+\infty} P_{x_{0}}^{1}\left(\sigma_{G_{k}}<+\infty\right)=+\infty$ if and only if $\sum_{k=1}^{+\infty} P_{x_{0}}^{1}\left(\sigma_{B_{k}}<+\infty\right)=+\infty$.

Before the proof of ii) of Theorem 5 we shall prepare two results from potential theory. Let $E$ be a bounded Borel set, and let us put

$$
V^{\varphi}(E)=\inf _{\mu \in L_{E}}\left\{\sup _{x \in R^{d}} \int_{\Omega} \varphi(|x-y|) \mu(d y)\right\},
$$

where $L_{E}=\{$ measure $\mu, S(\mu) \subset E, \mu(E)=1\}$. Then we define the $\varphi$-capacity $C^{\varphi}(E)$ of $E$ as follows;

1). $\quad V^{\varphi}(E)=+\infty \Longrightarrow C^{\varphi}(E)=0$ 
2). $\quad V^{\varphi}(E)<+\infty \Longrightarrow \varphi\left(C^{\varphi}(E)\right)=V^{\varphi}(E)$.

Lemma 1. (S. Kametani). Let $\varphi(t) \in \Phi_{1}$ and $h(t)=\frac{1}{\varphi(t)}$. Then, for each bounded Borel set $E, \Lambda^{h}(E)<+\infty$ means $C^{\varphi}(E)=0$, where $\Lambda^{h}(E)$ denotes $h$ Hausdorff measure of $E$.

For the proof, see S. Kametani [16]. (Also see S.J. Taylor [17].)

Lemma 2. [S.J. Taylor] Let $\varphi_{1}$ and $\varphi_{2}$ be functions of class $\Phi_{1}$ and $h(t)=\frac{1}{\varphi_{2}(t)} \cdot \quad$ Then, if

$$
\lim _{t \rightarrow 0+} \inf _{t} \frac{\varphi_{1}(t)}{\varphi_{2}(t)}=0
$$

holds, there exists a compact set $E$ such that $\Lambda^{h}(E)=0$ and $C^{\rho_{1}}(E)>0$.

For the proof see S.J.Taylor [17].

Lemma 3. Let $X^{\varphi}$ be a Feller process in $R^{d}$ whose Green function $G(x, y)=\varphi(|x-y|)$, where $\varphi(t) \in \Phi_{1}^{M}$ (see remark 1 in \$3). Then, for a compact set $K$, we have

$$
\mu_{K}^{\varphi}(K)=0 \Leftrightarrow C^{\varphi}(K)=0,
$$

where $\mu_{K}^{\varphi}(d y)$ denotes the capacitary measure of $K$ for $X^{\varphi}$.

Proof. If we assume that $\mu_{K}^{\varphi}(K)>0$, then the measure $\tilde{\mu}_{K}(d y)=\mu_{K}^{\varphi}(K)^{-1}$. $\mu_{K}^{\varphi}(d y)$ has total mass 1 and it holds $\int_{K} \varphi(|x-y|) \tilde{\mu}_{K}(d y) \leqq \frac{1}{\mu_{K}^{\varphi}(K)} \cdot$ Hence $V^{\varphi}(E)<+\infty$, which means $C^{\varphi}(K)>0$. Conversely, if we assume $C^{\varphi}(K)>0$, then there exists a measure $\mu$ whose support is in $K$ and $\int \varphi(|x-y|) \mu(d y)$ $\leqq V^{\varphi}(K)+\varepsilon<+\infty$ for each $\varepsilon>0$. Hence, if we set $\tilde{\mu}(d y)=\frac{\mu(d y)}{V^{\varphi}(K)}$, $\int \varphi(|x-y|) \mu(d y) \leqq 1+\varepsilon$ and support of $\tilde{\mu} \subset K$, which means $\mu_{K}^{\varphi}(K)>0$ by Lemma 5 in $\S 4$, because $X^{\varphi}$ satisfies the condition $(H)$ by Lemma 6 in $\S 4$.

Proof of ii) of theorem 5. By Taylor's theorem (Lemma 2) and Lemma 1 , there exists a compact set $K$ such that $C^{\varphi_{2}}(K)=0$ and $C^{\varphi_{1}}(K)>0$, which means $\mu_{K}^{\varphi_{2}}(K)=0$ and $\mu_{K}^{\varphi_{1}}(K) \neq 0$ by Lemma 3. Since $X^{\varphi_{1}}$ and $X^{\varphi_{2}}$ satisfy Hunt's condition $(H)$, some point is a regular point of $K$ for $X^{\varphi_{1}}$ but no point are regular points of $K$ for $X^{\varphi_{2}}$. Noting Theorem 5, i), the statement also holds for $X^{1}$ and $X^{2}$. 
ExAmple. Let $\mathscr{D}$ be a differential operator in $R^{d}(d \geqq 3)$ of the form

$$
\mathscr{D}=\sum_{i, j=1}^{d} a_{i j}(x) \frac{\hat{o}^{2}}{\partial x_{i} \partial x_{j}}+\sum_{i=1}^{d} b_{i}(x) \frac{\partial}{\partial x_{i}}-C(x) .
$$

Here $\mathscr{D}$ is assumed to be strictly elliptic, i.e.

$$
\mu^{-1} \sum_{i=1}^{d} \lambda_{i}^{2} \leqq \sum_{i, j=1}^{d} a_{i j}(x) \lambda_{i} \lambda_{j} \leqq \mu \sum_{i=1}^{d} \lambda_{i}^{2}, \mu>0
$$

and

$$
\left|b_{i}(x)\right|<M, \quad 0<C_{0} \leqq C(x) \leqq M, \lim _{\varepsilon \rightarrow 0} \sup _{x \in \Omega} \int_{0}^{\varepsilon} h^{-1} \cdot w(x, h) d h=0,
$$

where $M$ and $C_{0}$ are positive constants and $w(x, h)=\sup _{i \cdot j,|y|<h}\left|a_{i j}(x+y)-a_{i j}(x)\right|$. N.V. Krylov has shown that on a suitable domain $\Omega$ there exists a Feller process which is a quasi-diffusion Feller process connected with $\mathscr{D}$ having a Green function $G(x, y)$ with singularity $t^{2-a}$. (See N.V. Krylov [8], [9], Th. 4). Hence, by Theorem 5, a point is a regular point of a compact set for the above quasi-diffusion process, if and only if it is a regular point of the same set for the Brownian motion.

As another application of Theorem 5, we shall give a sufficient condition such that Hunt's hypothesis $(H)$ holds, making use of singularity function.

Proposition. Let $X$ be a Feller process which has the Green function $G(x, y)$ with singularity $\varphi \in \Phi_{1}^{M}$. Then $X$ satisfies the condition $(H)$, under the condition $(B)$.

Proof. Let $X^{\varphi}$ be a Feller process whose Green function $G^{\varphi}(x, y)=$ $\varphi(|x-y|)$. Then $X^{\varphi}$ satisfies the condition $(H)$ by Lemma 5 in $\S 4$ and, for each compact set, its regular point is also a regular point for $X$ and converse is true. Therefore we have only to show that, for each compact set $K, \mu_{K}(K)>0$ means $\mu_{K}^{\varphi}(K)>0$, where $\mu_{K}(d y)$ and $\mu_{K}^{\varphi}(d y)$ are the capacitary measures of $K$ for $X$ and $X^{\varphi}$ respectively. Let $\left\{Q_{n}\right\}$ be a sequence of nearly analytic sets such that $Q_{n}^{\text {co-reg }} \supset K$ and $\mu_{Q_{n}}^{\varphi}\left(\bar{Q}_{n}\right) \downarrow \mu_{K}^{\varphi}(K)$ (see Lemma 3 in $\$ 4$, then we have for sufficiently small $K$

$$
\begin{aligned}
\mu_{Q_{n}}^{\varphi}\left(\bar{Q}_{n}\right) & \geqq \int_{\Omega} P_{x}\left(\sigma_{K}<+\infty\right) \mu_{Q_{n}}^{\varphi}(d x) \\
& \geqq C \iint_{\Omega \times \Omega} \varphi(|y-x|) \mu_{K}(d y) \mu_{Q_{n}}^{\varphi}(d x)
\end{aligned}
$$




$$
\begin{aligned}
& =C^{\prime} \int P_{x}^{\varphi}\left(\sigma_{Q_{n}}<+\infty\right) \mu_{K}(d x) \\
& =C^{\prime} \mu_{K}(K), \quad C, \quad C^{\prime}>0,
\end{aligned}
$$

which means that $\mu_{K}(K)>0$ implies $\mu_{K}^{\varphi}(K)>0$.

\section{§8. A Green function with singularity $\left(\frac{1}{t}\right)^{3-\alpha}\left(\log \frac{1}{t}\right)$}

In this section we shall give an example of a kernel with singularity $\varphi$ satisfying the complete maximum principle which is not a type of Riesz kernel. For this purpose we use Deny's result [1]. Let $K$ be a measure in $R^{d}$. We say that $K$ satisfies the balayage principle, if for any compact set $C$ and for any measure $\mu$ of compact support, there exists a measure $\mu^{\prime}$ whose support is in $C$ such that

$$
\begin{array}{ll}
\left(B_{1}\right) \quad K * \mu^{\prime} \leqq K * \mu \quad \text { in } \quad R^{d} ; \\
\left(B_{2}\right) \quad K * \mu^{\prime}=K * \mu \text { on } \quad C \\
\left(B_{3}\right) \quad \int d \mu^{\prime} \leqq \int d \mu,
\end{array}
$$

where * denotes the convolution operator. The measure $\mu^{\prime}$ is called the balayaged measure of $\mu$ on $C$.

Lemma 1. (J. Deny). Let us set

$$
K=\mathscr{F}\left[\left(\int_{0}^{2}|y|^{s} \mu(d s)\right)^{-1}\right], y \in R^{d} \quad(d \geqq 3),
$$

where $\mu(d s)$ is a measure whose support is contained in the closed interval [0,2] and $\mathscr{F}$ denotes the Fourier transform in Schwartz's sense. Then $K$ is a measure which satisfies the balayage principle.

For the proof, see J. Deny [1].

Lemma 2. Let $K$ be a measure of function type $\varphi(x) d x$, where $\varphi(x)$ is a non-negative, locally integrable function such that $\varphi(x)=\varphi(|x|)$ and $d x$ is the Lebesgue measure. Then, if $K$ satisfies the balayage principle, it satisfies the complete maximum principle.

Proof. Let $f$ and $g$ be non-negative continuous functions with compact support such that the inequality

$$
K * f(x) \leqq K * g(x)+a
$$


holds on the support of $f$, where is a an arbitrary but fixed non-negative constant. We shall show that the above inequality holds everywhere. Let $\delta_{x}$ be a Dirac measure at $x$ and $\sigma$ be its balayaged measure on $S(f)(=$ support of $f$ ), then we have

$$
\begin{aligned}
K * f(x) & =\int_{R^{d}} f(y)\left(K * \delta_{x}\right)(d y)=\int_{R^{d}} f(y)(K * \sigma)(d y) \\
& =\int_{R^{d}} \sigma(d z) \int_{R^{d}} f(z+y) K(d y)=\int_{R^{d}} \sigma(d z) \int f(y) \varphi(|z-y|) d y \\
& \leqq \int_{R^{d}} \sigma(d z)\left\{\int_{R^{d}} \varphi(|z-y|) g(y) d y+a\right\} \leqq \int_{R^{d}} \varphi(|x-y|) g(y) d y+a .
\end{aligned}
$$

The proof is completed.

Let us now set

$$
K_{\alpha, k}=\mathscr{F}\left[\left(\int_{0}^{\alpha}|y|^{s} s^{k} d s\right)^{-1}\right], \quad 1<\alpha \leqq 2, \quad k ; \text { integer } \geqq 1 .
$$

In the 3-dimensional Euclidean space $R^{3}$ we can easily show that $K_{\alpha, k}$ is absolutely continuous with respect to the Lebesgue measure. Further, we can show that the density function $\psi$ such that $G(x, y)=\psi(|x-y|)$ is a kernel on $R^{3}$ with singularity $\left(\frac{1}{t}\right)^{3-\alpha}\left(\log \frac{1}{t}\right)$ which satisfies the complete maximum principle. Let us first note the following well-known result on Fourier transform: Let $f(x)$ be a measurable function on $R^{d}$ such that there exists $C>0$ and a positive integer $l$ and $|f(x)| \leqq C|x|^{l}$ holds for large $|x|$. Suppose further that $f(x)$ is only a function of $|x|$.

Then, it holds

$$
\mathscr{F} f(|x|)=\lim _{A \rightarrow \infty} \frac{2 \pi}{|x|^{\frac{d-2}{2}}} \int_{0}^{A} f(s) s^{\frac{d}{2}} J_{\frac{d-2}{2}}(2 \pi|x| s) d s, \quad\left(\text { in } \mathscr{S}^{\prime}\right),
$$

where $\frac{J_{\frac{d-2}{2}}}{2}$ is the Bessel function of order $\frac{d-2}{2}$. The next equality follows immediately from the above formula.

$$
K_{\alpha, K}=\lim _{A \rightarrow \infty} \frac{2}{|x|} \int_{0}^{A} \frac{r}{U_{k}(r)} \sin (2 \pi|x| r) d r, \quad 1<\alpha \leqq 2, \quad k>1,
$$

where

$$
U_{k}(r)=\int_{0}^{\infty} r^{s} s^{k} d s
$$

We divide the above integral into three parts as follows; 


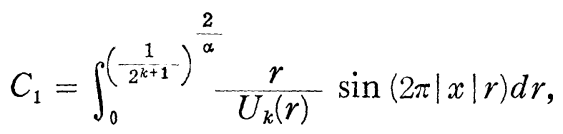

$$
\begin{aligned}
& C_{2}=\int_{\left(\frac{1}{2^{k+1}}\right)^{-\frac{2}{\alpha}}} e^{2 k k ! \cdot\left(\frac{1}{\alpha-1}\right)^{\frac{2}{\alpha-1}}} \frac{r}{U_{k}(r)} \sin (2 \pi|x| r) d r, \\
& C_{3}=\lim _{A \rightarrow \infty} \int_{e^{2 k k !} \cdot\left(\frac{1}{\alpha-1}\right)^{\alpha-1}}^{\frac{2}{U_{k}(r)}} \sin (2 \pi|x| r) d r .
\end{aligned}
$$

For $0<r<1$ we have

$$
k U_{k-1}(r)=k \int_{0}^{\alpha} r^{s} s^{k-1} d s \geqq k \int_{0}^{\frac{\alpha}{2}} r^{s} s^{k-1} d s \geqq r^{\frac{\alpha}{2}}\left(\frac{\alpha}{2}\right)^{k} .
$$

Moreover we have

$$
U_{k}(r)= \begin{cases}\frac{\alpha^{k} r^{\alpha}}{\log r}-\frac{k}{\log r} U_{k-1}(r) & r \neq 1 \\ \frac{\alpha^{k+1}}{k+1} & r=1 .\end{cases}
$$

Therefore we have for $r<\left(\frac{1}{2^{k+1}}\right)^{\frac{2}{\alpha}}<1$

$$
U_{k}(r)=\frac{k U_{k-1}(r)-\alpha^{k} r^{\alpha}}{\log \frac{1}{r}} \geqq \frac{1}{2^{k+1}} \alpha^{k} r^{\frac{\alpha}{2}} \frac{1}{\log \frac{1}{r}} .
$$

Hence it holds that

$$
C_{1} \leqq \frac{2^{k+1}}{\alpha^{k}} \int_{0}^{\left(\frac{1}{2^{k+1}}\right)^{\frac{2}{\alpha}}} r^{1-\frac{\alpha}{2}} \cdot \log \frac{1}{r} d r<K_{1},
$$

where $K_{1}$ is a positive constant which is independent of $x$.

It is clear that there exists a constant $K_{2}$ which is independent of $x$ such that

$$
\left|C_{2}\right|<K_{2}
$$

because $|\sin 2 \pi| x|r| \leqq 1$ and $\frac{r}{U_{k}(r)} \quad$ is continuous in $\left[\left(\frac{1}{2^{k+1}}\right)^{\frac{2}{\alpha}}, e^{2 k k !}\right.$. $\left.\left(\frac{1}{\alpha-1}\right)^{\frac{2}{\alpha-1}}\right]$. Now we shall estimate $C_{3}$. We shall first note that $\frac{U_{k}(r)}{r}$ 
is monotone increasing for $r>1$. Indeed, as we have for $0 \leqq s \leqq 1$ and $r \geqq 1$

$$
\frac{1-s}{(\alpha-1)^{2}} s^{k-1} \frac{1}{\{(\alpha-1) s+1\}^{k}} \leqq \frac{1}{(\alpha-1)^{2}}, r^{(\alpha-2) s+1} \geqq r^{\alpha-1},
$$

it holds for $r>\left(\frac{1}{\alpha-1}\right)^{\frac{2}{\alpha-1}} \cdot e^{2 k k !}$

(8. 2) $\frac{d}{d r}\left(\frac{U_{k}(r)}{r}\right)=\int_{0}^{\alpha}(s-1) r^{s-2} s^{k} d s$

$$
\begin{aligned}
&=\int_{0}^{\alpha-1} s r^{s-1}(s+1)^{k} d s-\int_{0}^{1}(1-s) r^{s-2} s^{k} d s \\
&=\int_{0}^{1} s(\alpha-1)^{2}\{(\alpha-1) s+1\}^{k} r^{s-2}\left\{r^{(\alpha-2) s+1}-\frac{1-s}{(\alpha-1)^{2}} s^{k-1} \times\right. \\
&\left.\times \frac{1}{\{(\alpha-1) s+1\}^{k}}\right\} d s
\end{aligned}
$$

$\geqq 0$.

Hence we have by the second mean value theorem

$$
C_{3} \leqq \frac{K_{3}}{|x|},
$$

where $K_{3}$ is a positive constant which is independent of $x$. Therefore we have

$$
\left|K_{\alpha, k}\right| \leqq \frac{K_{4}}{|x|}+\frac{K_{3}}{|x|^{2}}
$$

where $K_{4}$ is a positive constant independent of $x$. In the following we shall show that there exists a strictly positive constant $K_{5}$ such that

$$
\lim _{|x| \rightarrow 0} \frac{K_{\alpha, k}}{|x|^{\alpha-3}\left(\log \frac{1}{|x|}\right)}=K_{5}
$$

It is sufficient to show that

$$
\lim _{|x| \rightarrow 0} \frac{C_{3}}{|x|^{\alpha-2}\left(\log \frac{1}{|x|}\right)}=K_{5}
$$

By the change of the variable we have

$$
\int_{A}^{B} \frac{r}{U_{k}(r)} \sin 2 \pi|x| r d r=\int_{A|x|}^{B|x|} \frac{\frac{r}{|x|}}{U_{k}\left(\frac{r}{|x|}\right)} \cdot \sin (2 \pi r) \cdot \frac{d r}{|x|} .
$$


We shall first show that for any given $\varepsilon>0$ there exists a constant $A^{\prime}$ (independent of $|x|<\frac{1}{2}$ ) such that

$$
C_{x}^{A \prime}=\int_{A^{\prime}}^{A^{\prime \prime}} \frac{1}{|x|} \cdot|x|^{2-\alpha}\left(\log \frac{1}{|x|}\right)^{-1} \cdot \frac{\frac{r}{|x|}}{U_{k}\left(\frac{r}{|x|}\right)} \sin (2 \pi r) d r<\varepsilon
$$

for each $|x|<1$ and $A^{\prime \prime}>A^{\prime}$. If we choose $A^{\prime}>\left(\frac{1}{\alpha-1}\right)^{\frac{2}{\alpha-1}} e^{2 k k !} \geqq|x|$, $\frac{r}{|x|}\left(U_{k}\left(\frac{r}{x}\right)\right)^{-1}$ is a monotocically decreasing function of $r$ on $\left[A^{\prime},+\infty\right]$ by (8. 2). So by the second mean value theorem we have

$$
\left|C_{x}^{A \prime}\right| \leqq 2|x|^{-\alpha}\left(\log \frac{1}{|x|}\right)^{-1} \frac{A^{\prime}}{U_{k}\left(\frac{A^{\prime}}{|x|}\right)} .
$$

Let us note that

$$
U(r)=\left\{\begin{array}{lc}
\frac{r^{\alpha}}{\log r} \sum_{l=0}^{k}\left\{\alpha^{k-l}(-1)^{l} \frac{1}{(\log r)^{l}} \frac{k !}{(k-l) !}\right\}+(-1)^{k+1} k ! \frac{1}{(\log r)^{k+1}} \\
-\frac{\alpha^{k}}{k+1} & r \neq 1 \\
0 & r=1 \\
& r=0 .
\end{array}\right.
$$

Indeed, using the equality (3.1), we can get the above equality by the induction. Therefore we have for $2 \geqq \alpha>1$

$$
\begin{gathered}
\mid C_{x}^{A^{\prime} \mid} \leqq 2 A^{\prime 1-\alpha} \cdot\left(\frac{\log \frac{A^{\prime}}{|x|}}{\log \frac{1}{|x|}}\right) \cdot \frac{\frac{1}{k !}}{\left\{\sum_{l=0}^{k} \alpha^{k-l}(-1)^{l}\left(\log \frac{A^{\prime}}{|x|}\right)^{-l} \cdot \frac{k !}{(k-l) !}\right\}+} \\
\leqq 2 A^{\prime 1-\alpha}\left(1+\frac{\log A^{\prime}}{\log \frac{1}{|x|}}\right) \cdot\left\{\left\{\sum_{l=0}^{k} \alpha^{k-l}(-1)^{l}\left(\log \frac{A^{\prime}}{|x|}\right)^{-l} \frac{k !\left(\frac{x}{A^{\prime}}\right)^{\alpha} \cdot\left(\log \frac{A^{\prime}}{|x|}\right)^{-k}}{(k-l)}\right\}+(-2 k !)\left(\frac{1}{A^{\prime}}\right)\right\}^{-1}
\end{gathered}
$$

Hence, if we choose $A^{\prime}$ such that $\frac{\alpha^{k}}{2}>\sum_{l=1}^{k} \alpha^{k-l}\left(\log A^{\prime}\right)^{-l} \cdot k !+2 k !\left(\frac{1}{A^{\prime}}\right)^{\alpha}$, it holds for $|x|<\frac{1}{2}$ 


$$
\begin{aligned}
\left|C_{x}^{A^{\prime}}\right| & \leqq 4 A^{\prime 1-\alpha}\left(1+\frac{\log A^{\prime}}{\log \frac{1}{|x|}}\right) \cdot \frac{1}{\alpha^{k}} \\
& \leqq 4 A^{\prime 1-\alpha}\left(1+(\log 2)^{-1} \log A^{\prime}\right) \cdot \frac{1}{\alpha^{k}} .
\end{aligned}
$$

Hence (8. 5) holds for sufficiently large $A^{\prime}$. In the following we shall fix such $A^{\prime}$, and show

$$
\begin{aligned}
& \lim _{|x| \rightarrow 0} \int_{\left(\frac{1}{\alpha-1}\right)^{-\frac{2}{\alpha-1}} e^{2 k k ! \cdot|x|}}|x|^{2-\alpha}\left(\log \frac{1}{|x|}\right)^{-1} \cdot \frac{1}{|x|} \frac{\frac{r}{|x|}}{U_{k}\left(\frac{r}{|x|}\right)} \sin (2 \pi r) d r \\
& =\int_{0}^{A^{\prime}} \frac{1}{\alpha^{k}} r^{1-\alpha} \sin 2 \pi r d r>0 .
\end{aligned}
$$

The left-hand side of (8.6) equals to

$$
\begin{aligned}
\lim _{|x| \rightarrow 0} \int_{\left(\frac{1}{\alpha-1}\right)^{A^{\prime}}}^{\frac{2}{\alpha-1}} e^{2 k k ! \cdot|x|}\left(1-\frac{\log \frac{1}{r}}{\log \frac{1}{|x|}}\right) \cdot r^{1-\alpha} \sin (2 \pi r) \cdot \\
\\
\left\{\sum_{l=0}^{k} \alpha^{k-l}\left(\log \frac{r}{|x|}\right)^{-l} \frac{1}{\left.(-1)^{l} \frac{k !}{(k-l) !}\right\}+(-1)^{k+1} k !\left(\frac{|x|}{r}\right)^{\alpha}\left(\log \frac{r}{|x|}\right)^{-k}} d r .\right.
\end{aligned}
$$

Noting that

$$
\begin{aligned}
(*) & =\sum_{l=0}^{k} \alpha^{k-l}\left(\log \frac{r}{|x|}\right)^{-l}(-1)^{l} \frac{k !}{(k-l) !}+(-1)^{k+1} k !\left(\frac{|x|}{r}\right)^{\alpha}\left(\log \frac{r}{|x|}\right)^{-k} \\
& \geqq\left(\log \frac{r}{|x|}\right)^{-k}\left\{\alpha^{k}\left(\log \frac{r}{|x|}\right)^{k}-\alpha^{k}\left(\log \frac{r}{|x|}\right)^{k-1} k \cdot k !\right\}-\frac{\left(\frac{|x|}{r}\right)^{\alpha}}{\left(\log \frac{r}{|x|}\right)^{k}} \cdot k ! \\
& \geqq \frac{1}{2} \alpha^{k}-k ! \cdot \frac{\left(\frac{|x|}{r}\right)^{\alpha}}{\left(\log \frac{r}{|x|}\right)^{k}} \geqq \frac{1}{4} \alpha^{k}>0 \text { for } A^{\prime}>r>e^{2 k k^{\prime}}|x|,
\end{aligned}
$$

we see that

$$
\begin{aligned}
& \left|\chi_{\left[e^{\left.2 k k ! \cdot|x|, A^{\prime}\right]}\right.}\left(1-\frac{\log \frac{1}{r}}{\log \frac{1}{|x|}}\right) r^{1-\alpha} \cdot \sin 2 \pi r \cdot \frac{1}{(*)}\right| \\
& \leqq K_{6}
\end{aligned}
$$


where $K_{6}$ is independent of $x$ and $r$ and $\chi_{\left[e^{2 k k !}|x|, A^{\prime}\right)}$ is a characteristic function of $\left[e^{2 k k !}|x|, A^{\prime}\right)$. Hence we can obtain the equality (8.6). We can also show that $K_{\alpha, k}$ is continuous except at the origin, so it is nonnegative because it is positive almost everywhere. (Remark that $K_{\alpha, k}$ is a measure.) Hence $K_{\alpha, k}$ is a kernel with singularity $\left(\frac{1}{t}\right)^{3-\alpha}\left(\log \frac{1}{t}\right)$ which satisfies the complete maximum principle.(Note Lemma 2.)

\section{REFERENCES}

[ 1 ] J. Deny, Le Balayage, Meddel. Lunds Univ. Mat. Sem., tome dedié à M. Riesz (1952), $47-61$.

[2] E.B. Dynkin, Markov process, Springer-Verlag, Berlin.

[ 3 ] O. Frostman, Potentiel d'equilibre et capacité des ensembles avec quelques applications à la theorie des fonctions, Meddel. Lunds Univ. Mat. Sem. 3 (1935).

[4] G.A. Hunt, Mrkoff processes and potentials. 3, Illinois J. Math. (1958), 151-213.

[5] K. Ito and H.P. Mckean. Jr, Diffusion processes and their sample paths, SpringerVerlag, 1965.

[6] M. Kanda, Regular points and Green functions in Markov processes, J. Math. Soc. Japan, Vol. 19, No. 1, (1967), 46-69.

[ 7 ] M. Kanda, A remark on the continuity of the dual process, Nagoya Math. J. Vol. 32, (1968), 287 295.

[8] N.V. Krylov, On quasi-diffusional processes, Theorey Probability Appl. (USSR) (1966), 424-443.

[9] N.V. Krylov, On the green function for the Dirichlet problem, Uspehi Mat. Nauk, Tom. 22. No. 2 (134) (1967), 116-118.

[10] H. Kunita and T. Watanabe, Markov processes and Martin boundaries, Illinois J. Math., 9 (1965), 485-526.

[11] P.A. Meyer, Proprietes des fonctions excessives, Séminaire Brelot-Choquet-Deny (théorie du potentiel), (1960-1961).

[12] P.A. Meyer, Semi-groupes en dualité, Séminaire Brelot-Choquet-Deny (théorie du potentiel), (1960-1961).

[13] P.A. Meyer, Fonctionelles multiplicatives et additives de Markov, Ann. Inst. Fourier, 12 (1962).

[14] M.G. Sur, Martin boundary for linear elliptic operators of second order, Izv. Akad. Nauk (USSR), 27 (1963), 45-60.

[15] S. Watanabe, J. Takeuchi and T. Yamada, Stable process, Sem. on Prob. Vol. 13, (in Japanese).

[16] S. Kametani, On Hausdorff's measures and generalized capacities with some of their applications to the theory of functions. Japanese J. Math. 19 (1944), 217-257.

[17] S.J. Taylor, On the connection between Hausdorff measures and generalized capasity. Proc. Cambridge Philos. Soc., 57 (1961), 524-531.

Nagoya University. 


\section{SUPPLEMENT}

It is clear that the theorem 1 also holds under the condition $B$ for the Feller process on $\Omega$ which hsa a Green function $G(x, y)$ such that, for each ball $O_{n} \subset \Omega$ with radius $\frac{1}{n}, \lim _{n \rightarrow \infty}\left(\inf _{(x, y) \in O_{n} \times O_{n}} G(x, y)\right)=+\infty$. The proof is the same as that of theorem 1 .

Here, by the condition $B$, we mean

$$
P_{x}\left(\sigma_{K}<+\infty\right)=P_{x}\left(\sigma_{K}\left(w_{\sigma_{G}}^{+}\right)<+\infty\right)
$$

holds for $x \in \Omega-\bar{G}$, where $K$ (resp. G) is a nearly analytic set (resp. open set) with compact closure in $\Omega$ such that $\bar{K} \subset G$. For example, a continuous Feller process satisfies the condition $B$. 